\title{
The impact of COVID-19 on the provision of donor hematopoietic stem cell products worldwide: collateral damage
}

\author{
Jeff Szer $\mathbb{1}^{1} \cdot$ Daniel Weisdorf $^{2} \cdot$ Sergio Querol $^{3} \cdot$ Lydia Foeken $^{4} \cdot$ Alejandro Madrigal $^{5}$
}

Received: 5 March 2020 / Revised: 9 March 2020 / Accepted: 12 March 2020 / Published online: 23 March 2020

(c) Springer Nature Limited 2020

\section{To the Editor:}

The provision of donors for life-saving hematopoietic stem cell transplants is coordinated through registries and facilitated by the World Marrow Donor Association (WMDA), which operates a global database to find the best stem cell source. The database contains 36,214,535 donors and 790,357 cord blood units from 98 different registries in 53 different countries [1].

The currently accelerating rate of COVID-19 infection and the responses of governments and facilities have the potential to impact and interfere with the timely provision of cells that must cross national borders. These cells are usually transported fresh after collection by individual or commercial couriers, while cord blood cells are transported while frozen though they still must be delivered promptly. In addition to transportation difficulties, possible exposures of the donor may affect the safety of the stem cell product. It is currently uncertain whether COVID-19 is transmissible parenterally, but it seems prudent to defer donors from endemic areas until suitable testing and observation time can ensure the safety of the product. This is particularly important if the donor is in a

\section{Jeff Szer}

Jeff.Szer@mh.org.au

1 Clinical Haematology at Peter MacCallum Cancer Centre and The Royal Melbourne Hospital, Melbourne, VIC, Australia

2 Blood and Marrow Transplant Program and Masonic Cancer Center, University of Minnesota, Minneapolis, MN, USA

3 Frederic Duran i Jordà Center, Catalan Blood and Tissue Bank (BST), Barcelona, Spain

4 World Marrow Donor Association, Schipholweg 57, 2316 ZL Leiden, The Netherlands

5 Anthony Nolan Research Institute Royal Free Hospital \& UCL Medical School, London, UK country or region with a high rate of COVID-19 infection. Donation may be precluded by available health resources diverted to the infection, perceived risks to couriers and the consequent delays in exporting and importing the product.

A number of strategies have been proposed to assist in navigating through these rapidly evolving difficulties [2-4]. Timely communication to donors and patients with strategies to assist in the provision of alternative products and procedures to optimize cell collections may be required. Pre-emptive cryopreservation of products that are likely to be required at a time when transport may be further restricted is one option. This would allow complete infectious testing to ensure that the product is not contaminated by infectious agents before use.

In addition, there may a need to consider cord blood products more frequently as these products will have been collected and stored before the current infection became evident and cord blood units are always available immediately.

Policy and practice considerations in these unprecedented circumstances are mandatory and registries need to work with responsible authorities to minimize interference with necessary medical treatment and facilitate any available alternatives when required.

Organizations like WMDA could be vital to coordinate the urgent needs for cellular products. International cooperation and well-coordinated measures for donor provision will ensure that patients will be treated with the best product at the appropriate time.

\section{Compliance with ethical standards}

Conflict of interest The authors declare that they have no conflict of interest.

Publisher's note Springer Nature remains neutral with regard to jurisdictional claims in published maps and institutional affiliations. 


\section{References}

1. https://statistics.wmda.info Accessed 4 March 2020.

2. https://share.wmda.info/x/ED6OF Accessed 4 March 2020.

3. https://share.wmda.info/x/Yj6OF Accessed 4 March 2020.
4. https://share.wmda.info/display/LP/Coronavirus +-+Impact+on + Registry + Operations?preview $=/ 344866402 / 344867270 /$ WBMT_COVID-19-2.pdf\#Coronavirus-ImpactonRegistryOpera tions-WorldwideNetworkForBlood\&MarrowTransplantation (WBMT) Accessed 4 March 2020. 